\title{
BISFOSFONATOS: SÍNTESE, ANÁLISES QUÍMICAS E APLICAÇÕES FARMACOLÓGICAS
}

\author{
Christian Fernandes, Rodrigo Souza Leite e Fernando Mauro Lanças* \\ Departamento de Química e Física Molecular, Instituto de Química de São Carlos, Universidade de São Paulo, CP 780, \\ 13560-970 São Carlos - SP
}

Recebido em 22/1/04; aceito em 13/8/04; publicado na web em 2/2/05

\begin{abstract}
BISPHOSPHONATES: SYNTHESIS, CHEMICAL ANALYSIS AND PHARMACOLOGICAL APPLICATIONS. Bisphosphonates are drugs that have been widely used in different bone diseases, and have recently been used successfully against many parasites. Various synthetic routes to prepare different types of bisphosphonates have been described, with distinct potency and pharmacological activity. A number of analytical techniques are currently being used to analyze these drugs; among these, the high performance liquid chromatography (HPLC), with different systems of detection, is worth highlighting. However, the development of more sensitive methods is still necessary, once they are essential for bioavailability and bioequivalence studies. This paper reports the major synthesis routes, chemical analysis methodologies and pharmacological applications of bisphosphonates.
\end{abstract}

Keywords: bisphosphonates; bone diseases; high performance liquid chromatography.

\section{INTRODUÇÃO}

Os bisfosfonatos (BP's) formam uma classe de substâncias químicas que apresenta uma ligação P-C-P em sua estrutura, e agem como inibidores da reabsorção óssea, mediada pelos osteoclastos. Estes compostos são extensivamente utilizados no tratamento de várias doenças ósseas, destacando-se a doença de Paget, a hipercalcemia maligna, a osteoporose e a doença metastática e osteolítica.

São análogos químicos da substância endógena denominada ácido pirofosfórico (Figura 1), que no organismo se encontra como pirofosfato, um inibidor natural da reabsorção óssea. No entanto, essa substância não pode ser utilizada como agente terapêutico no tratamento de doenças ósseas, pois sofre uma rápida hidrólise enzimática. Os bisfosfonatos são seus análogos sintéticos, onde o átomo central de oxigênio é substituído por um de carbono. Essa modificação faz com que os BP's sejam mais resistentes à degradação enzimática, e possuam uma meia-vida biológica maior, suficiente para influenciar o metabolismo ósseo ${ }^{1}$.

$$
\begin{array}{cc}
\mathrm{O} & \mathrm{O} \\
\text { II } & \text { II } \\
\mathrm{HO}-\mathrm{P}-\mathrm{O}-\mathrm{P}-\mathrm{OH} & \mathrm{I} \\
\mathrm{OH} & \mathrm{O} \\
\mathrm{OH} &
\end{array}
$$

Figura 1. Estrutura química do ácido pirofosfórico

Diferentes substituintes ligados ao carbono central dão características únicas para cada fármaco (Figura 2). O grupo R1 fornece a afinidade dos BP's pelos cristais ósseos, enquanto o grupo R2 é responsável pela potência e atividade farmacológicas ${ }^{2}$. Segundo Shinoda ${ }^{3}$, a adição de um grupo hidroxila ao átomo de carbono na posição 1 aumenta a potência anti-reabsortiva. Derivados com um grupo amino no final da cadeia lateral são extremamente ativos. Além disso, o comprimento da cadeia lateral é também muito importante, sendo a maior atividade encontrada em compostos com quatro carbonos, como o alendronato. Mais recentemente tem sido demonstrado que o grupo amino não tem que estar localizado necessariamente

*e-mail: flancas@iqsc.usp.br no fim da cadeia. No entanto, ainda não está bem clara a relação existente entre estrutura e atividade, podendo-se dizer apenas que a estrutura P-C-P é fundamental para a atividade e que a intensidade do efeito é intensamente dependente da cadeia lateral.

$$
\begin{array}{ccc}
\mathrm{O} & \mathrm{R}_{1} & \mathrm{O} \\
\text { II } & 1 & \mathbb{1} \\
\mathrm{HO}-\mathrm{P}-\mathrm{C} & \mathrm{P}-\mathrm{OH} \\
\text { I } & \mathrm{I} & \mathrm{I} \\
\mathrm{OH} & \mathrm{R}_{2} & \mathrm{OH}
\end{array}
$$

Figura 2. Estrutura química geral dos BP's

Os BP's são sintetizados por meio de uma grande variedade de rotas sintéticas. Estas levam à produção de BP's com diferentes estruturas químicas e rendimentos.

Os BP's podem ser analisados quantitativa e qualitativamente por um conjunto de técnicas analíticas eficientes. As mais usuais são a cromatografia líquida de alta eficiência (HPLC), cromatografia gasosa (GC) e eletroforese capilar (CE), utilizando diferentes detectores, tais como espectrômetro de massas (MS), índice de refração, espectrofotômetro de ultravioleta e eletroquímicos.

O objetivo do presente trabalho é mostrar a importância dos BP's no tratamento de várias doenças ósseas e suas novas aplicações. Propõe-se também descrever as rotas de síntese mais usuais, bem como os diferentes métodos utilizados na análise destes fármacos.

\section{FARMACOLOGIA}

\section{Farmacodinâmica}

O tecido ósseo contém três tipos de células: osteoblastos, osteoclastos e osteócitos. Os osteoblastos formam os ossos por deposição de materiais protéicos na matriz, levando à mineralização. A função dos osteoclastos é a reabsorção óssea. Essas células destroem a matriz sempre que o osso precisa ser remodelado. O terceiro tipo de células, os osteócitos, encontram-se localizados profundamente nas fibras protéicas da matriz óssea estando, provavelmente, envolvidos na homeostase do cálcio plasmático ${ }^{4}$.

O mecanismo molecular pelo qual os BP's inibem a reabsorção óssea ainda não é completamente entendido. Inicialmente, acredita- 
va-se que apenas um mecanismo físico-químico, após a adsorção óssea, era suficiente para sua ação $0^{5}$. No entanto, ultimamente, temse tornado claro que efeitos celulares também estão envolvidos ${ }^{5}$. Como os osteoclastos produzem endocitose, os BP's presentes no espaço onde ocorre a reabsorção podem penetrar nestas células, afetando seu metabolismo intracelular e induzindo apoptose ${ }^{5}$. Recentes estudos têm demonstrado que parte da ação inibitória dos BP's sobre os osteoclastos é mediada por uma atividade dos osteoblastos 6 .

Green $^{7}$ demonstrou em estudos in vitro que os BP's também inibem a proliferação, reduzem a viabilidade e induzem apoptose em muitas células tumorais humanas.

As propriedades físico-químicas dos BP's são bem similares entre os membros da série. No entanto, suas atividades anti-reabsortivas diferem grandemente. Por causa dessa grande diferença de potência entre os BP's, suas doses clínicas também diferem acentuadamente ${ }^{8}$. Os principais BP's estudados estão apresentados na Tabela 1.

Tabela 1. BP's e suas respectivas potências anti-reabsortivas relativas em modelos in vivo

\begin{tabular}{llll}
\hline Bisfosfonato & $\mathrm{R} 1$ & $\mathrm{R} 2$ & Potência \\
\hline Clodronato & $\mathrm{Cl}$ & $\mathrm{Cl}$ & $\sim 10$ \\
Etidronato & $\mathrm{OH}$ & $\mathrm{CH}_{3}$ & $\sim 1$ \\
Pamidronato & $\mathrm{OH}$ & $\left(\mathrm{CH}_{2}\right)_{2} \mathrm{NH}_{2}$ & $\sim 100$ \\
Alendronato & $\mathrm{OH}$ & $\left(\mathrm{CH}_{2}\right)_{3} \mathrm{NH}_{2}$ & $>100-<1000$ \\
Neridronato & $\mathrm{OH}$ & $\left(\mathrm{CH}_{2}\right)_{5} \mathrm{NH}_{2}$ & $\sim 100$ \\
Olpadronato & $\mathrm{OH}$ & $\left(\mathrm{CH}_{2}\right)_{2} \mathrm{~N}_{2}\left(\mathrm{CH}_{3}\right)_{2}$ & $>100-<1000$ \\
Ibandronato & $\mathrm{OH}$ & $\left(\mathrm{CH}_{2}\right)_{2} \mathrm{~N}_{(}\left(\mathrm{CH}_{3}\right)\left(\mathrm{CH}_{2}\right)_{4} \mathrm{CH}_{3}$ & $>1000-<10000$ \\
Risedronato & $\mathrm{OH}$ & $\mathrm{CH}_{2}-3$-piridina & $>1000-<10000$ \\
Zoledronato & $\mathrm{OH}$ & $\mathrm{CH}_{2}$-imidazol & $>10000$ \\
\hline
\end{tabular}

Os BP's tornaram-se uma alternativa para o tratamento de uma variedade de doenças ósseas onde há uma excessiva atividade dos osteoclastos, incluindo doença de Paget, hipercalcemia maligna, osteoporose e doença metastática e osteolítica ${ }^{9}$. São também úteis em muitas formas de osteoporose secundária, causadas por utilização de glicocorticóides, gravidez, transplante de órgãos e paraplegia ${ }^{1}$.

Altas doses de muitos BP's podem dificultar a mineralização normal dos ossos ${ }^{10}$. Dessa forma, busca-se atualmente o desenvolvimento de BP's com alta atividade anti-reabsortiva, que tenham uma ampla faixa de segurança para a mineralização normal.

O Trypanosoma cruzi (protozoário causador da doença de Chagas) contém importantes depósitos de pirofosfato e polifosfatos em organelas denominadas acidocalcisomas. Estudos recentes mostram que os BP's inibem o crescimento do protozoário T. cruzi in vitro e in vivo, por meio da competição com o pirofosfato em sua via metabólica, sem causar toxicidade nas células hospedeiras ${ }^{11}$.

Martin e colaboradores ${ }^{12}$ verificaram a ação dos BP's que possuem nitrogênio na cadeia lateral, sobre o crescimento dos parasitas Trypanosoma brucei, Trypanosoma cruzi, Leishmania donovani, Toxoplasma gondii e Plasmodium falciparum. O mecanismo de ação dos BP's consiste, basicamente, na inibição da formação de proteínas de importância vital para os parasitas.

A artrite reumatóide está relacionada com uma erosão óssea nas articulações. Alguns estudos clínicos têm sido realizados para avaliar a eficiência de agentes anti-artríticos; recentemente, a família dos BP's vem obtendo destaque no tratamento dessa doença ${ }^{13}$. Os ésteres dos BP's têm conseguido diminuir, significativamente, as inflamações associadas com a formação do granuloma cutâneo e com a artrite erosiva, inibindo a erosão óssea nas articulações ${ }^{13,14}$.

Devido à sua capacidade quelante, os bisfosfonatos também têm sido estudados para elaboração de novos medicamentos capazes de controlar a quantidade de íons ferro e alumínio no organismo huma- no ${ }^{15}$. Recentemente novos BP's foram sintetizados por meio das reações de Michaelis-Arbuzov, Michaelis-Becker e transesterificação do fosfonoacetato de trietila. Estas metodologias foram satisfatórias, tendo em vista o bom rendimento obtido e a facilidade de execução. Estes bisfosfonatos mostraram-se eficientes agentes quelantes frente a diferentes metais ${ }^{16}$.

Estudos realizados com etidronato e clodronato demonstraram que estes fármacos apresentam baixa toxicidade. Testes realizados em diferentes espécies animais indicaram não serem teratogênicos, mitogênicos e carcinogênicos ${ }^{17}$.

De maneira geral, os BP's apresentam poucos efeitos adversos. O etidronato pode causar inibição na mineralização normal dos ossos, originando fraturas ${ }^{18}$. Distúrbios gastrintestinais e alterações no paladar também podem ocorrer ${ }^{19}$. Para o clodronato, apenas alguns poucos casos de diarréia branda foram relatados ${ }^{20}$. Pamidronato pode causar distúrbios gastrintestinais tais como náusea, vômito e diarréia. Após a administração oral ou intravenosa, pode induzir pirexia de 1 a $2^{\circ} \mathrm{C}^{21}$.

\section{Farmacocinética}

A biodisponibilidade oral, fração de uma dose que alcança a circulação sistêmica, é determinada pela comparação da área total sob a curva de concentração no plasma versus tempo, após a administração oral e intravenosa ${ }^{22}$.

Os BP's apresentam baixa absorção intestinal em humanos, com biodisponibilidade de cerca de $0,7 \%$ para alendronato, $0,3 \%$ para pamidronato, 3-7\% para etidronato e $1-2 \%$ para clodronato ${ }^{8}$. Esta baixa absorção ocorre porque estes fármacos são pouco lipofílicos, dificultando o transporte através da barreira epitelial ${ }^{23}$. Além do mais, são moléculas relativamente grandes, negativamente carregadas no $\mathrm{pH}$ intestinal e complexam facilmente com o cálcio, prejudicando ainda mais a sua absorção ${ }^{24}$. Os alimentos causam uma acentuada diminuição na absorção dos BP's, devendo estes serem administrados pelo menos 30 min antes da primeira alimentação diária ${ }^{25}$.

Estudos realizados em ratos, com o alendronato, demonstraram que ele possui forte interação com as proteínas plasmáticas, principalmente a albumina sérica. $\mathrm{O}$ aumento do $\mathrm{pH}$ e da concentração de íons cálcio acentua ainda mais essa interação ${ }^{26}$.

Após a administração intravenosa, o alendronato é amplamente distribuído por todo o corpo, incluindo os tecidos calcificados e não calcificados. Os BP's ligam-se preferencialmente em ossos com alta taxa de remodelagem, devido à grande exposição da hidroxiapatita nesses sítios ${ }^{8}$.

Não foram encontradas evidências de metabólitos dos BP's nos estudos realizados, indicando serem muito estáveis. Essa característica minimiza a toxicidade do tratamento, devido à ausência de intermediários reativos e metabólitos tóxicos ${ }^{27}$.

A excreção renal é a única rota de eliminação dos BP's. Estudos realizados em animais e humanos indicam que os BP's administrados sistemicamente são parcialmente ligados aos tecidos ósseos e o restante é excretado pelos rins. Após a administração intravenosa, os BP's desaparecem do plasma rapidamente, com uma meia-vida de 1-2 h. Por outro lado, uma vez ligado ao osso, eles só são liberados quando o tecido ósseo, ao qual estão ligados, sofre reabsorção ${ }^{8}$.

\section{SÍNTESE}

O primeiro bisfosfonato utilizado no tratamento de doenças ósseas foi o etidronato, o qual pode ser facilmente sintetizado por meio de reação entre anidrido acético e ácido fosforoso (Esquema 1$)^{28}$.

Estudos posteriores demonstraram que os BP's que possuem um átomo de nitrogênio em sua cadeia lateral possuem potência supe- 


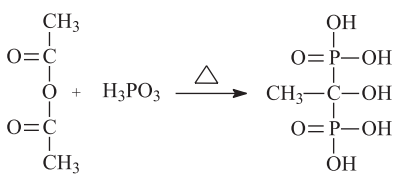

Esquema 1. Síntese do etidronato

rior ao etidronato ${ }^{29}$. Mais recentemente, foi demonstrado que o grupo amino não deve estar, necessariamente, no final da cadeia. A atividade é aumentada quando um grupo metil e um pentil são ligados ao nitrogênio ${ }^{30}$.

A reação mais comum utilizada na preparação desses BP's é entre um ácido carboxílico e o ácido fosforoso, na presença de tricloreto de fósforo (Esquema 2) ${ }^{30}$. Vários tipos de BP's são obtidos a partir de diversos ácidos carboxílicos, com bons rendimentos (Tabela 2). Os reagentes são deixados sob agitação a $65^{\circ} \mathrm{C}$ por aproximadamente $18 \mathrm{~h}$, utilizando como solvente o ácido metanossulfônico ou benzenossulfônico. Logo após, é adicionado hidróxido de sódio para ocorrer a precipitação dos sais de BP's.

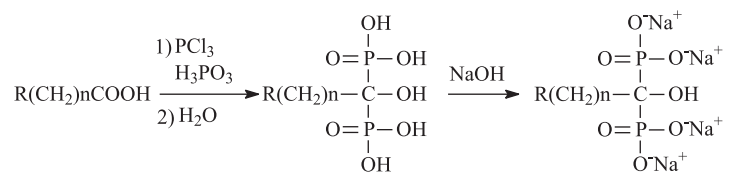

Esquema 2. Reação entre ácido carboxílico e ácido fosforoso na presença de tricloreto de fósforo

Tabela 2. Valores típicos de rendimento da síntese dos BP's para diferentes ácidos carboxílicos

\begin{tabular}{lcc}
\hline $\mathrm{R}$ & $\mathrm{n}$ & $\mathrm{R} \%$ \\
\hline $\mathrm{NH}_{2}$ & 2 & 57 \\
$\mathrm{NH}_{2}$ & 3 & 89 \\
$\mathrm{NH}_{2}$ & 4 & 78 \\
$\mathrm{NH}_{2}$ & 5 & 89 \\
$\mathrm{CH}_{3}$ & 10 & 95 \\
3-imidazol & 1 & 31 \\
3-piridil & 1 & 38 \\
4-aminofenil & 1 & 26 \\
$\mathrm{Cl}$ & 3 & 30
\end{tabular}

Esta síntese também pode ser realizada utilizando-se ácidos graxos de cadeia pequena e que não contenham nitrogênio (Esquema 3). Os BP's assim formados mostraram-se ativos contra o crescimento do Trypanosoma cruzi ${ }^{11}$.

Outra rota de síntese mais moderna é realizada pela reação entre um cloreto de acila e o tris(trimetilsilil)fosfito, com posterior metanólise ${ }^{31}$.

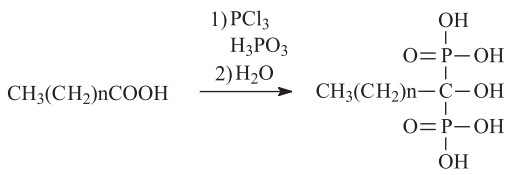

Esquema 3. Síntese de BP's a partir de ácidos graxos de cadeia pequena (n $=1,2,3$ e 4 )

A reação do cloreto de acila com 2 equivalentes de tris(trimetilsilil)fosfito leva, em poucos minutos e com temperatura de $25{ }^{\circ} \mathrm{C}$, à formação do éster tetrakis(trimetilsilil) do ácido 1trimetilsiloxi-1,1-bifosfônico. Logo após, ocorre a metanólise durante $1 \mathrm{~h}$ à temperatura de $25^{\circ} \mathrm{C}$. Os BP's são obtidos com a evaporação da fração volátil (Esquema 4).

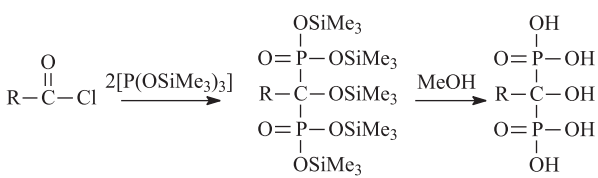

Esquema 4. Síntese de BP's por meio da reação entre o cloreto de acila e o tris(trimetilsilil)fosfito

Esta reação possui inúmeras vantagens quando comparada à reação mencionada anteriormente, destacando-se: condições mais brandas do sistema reacional, maiores rendimentos obtidos e menores tempos de reação. Por outro lado, observam-se algumas desvantagens: alto preço do reagente tris(trimetilsilil)fosfito e dificuldade de se encontrar comercialmente diferentes cloretos de acila (sendo necessária a preparação dos mesmos) ${ }^{31}$.

A preparação de cloretos de acila é bastante conhecida na literatura e consiste na reação entre um ácido carboxílico e o cloreto de tionila (Esquema 5) ${ }^{32}$. Entretanto, isso representa uma nova etapa na síntese dos BP's, acarretando, principalmente, redução do rendimento total.

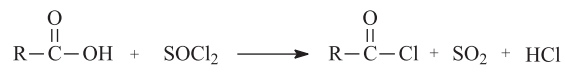

Esquema 5. Síntese de cloretos de acila

Outro método de preparação dos BP's consiste na reação de bishidrofosforilação de alcinos terminais por fosfitos de dialquila, catalisada por paládio ${ }^{33}$. Os alcinos terminais são sintetizados por uma reação de Heck entre um haleto de arila e o trimetilsililacetileno, com a remoção do grupo trimetilsilil em condições básicas (Esquema 6a). Os fosfitos de dialquila, por sua vez, são sintetizados pela reação entre um equivalente de tricloreto de fósforo e três equivalentes do álcool correspondente (Esquema $6 \mathrm{~b})^{34}$. Refluxando a mistura contendo um equivalente do alcino terminal e três equivalentes de fosfito de dialquila, na presença de $\mathrm{Pd}\left(\mathrm{PPh}_{3}\right)_{4}$ e tolueno (solvente), obtém-se uma grande variedade de BP's vicinais com ótimos rendimentos (Esquema 6c) ) $^{33}$.

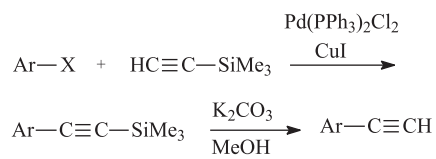

(a)

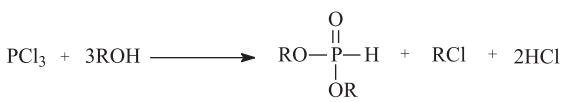

(b)

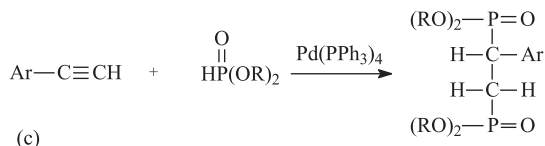

Esquema 6. Síntese de BP's através de uma reação de bis-hidrofosforilação catalisada por paládio: (a) síntese do alcino terminal; (b) síntese do fosfito de dialquila; (c) reação do alcino terminal com fosfito de dialquila 
Outros tipos de BP's podem ser preparados utilizando os íons enolato como intermediários. O tratamento de compostos carbonílicos com uma base forte e posterior adição de excesso de grupos eletrofílicos $\left[\mathrm{ClP}(\mathrm{OEt})_{2}\right]$ resulta na formação de duas ligações P-C no carbono $\alpha$. Estes BP's são obtidos após oxidação com peróxido de hidrogênio ${ }^{35}$. O Esquema 7 ilustra uma reação realizada nestas condições.

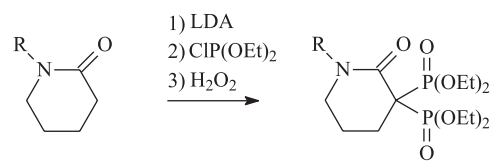

Esquema 7. Síntese de BP's utilizando íons enolato como intermediários

Na Tabela 3 são mostrados outros exemplos de produtos formados a partir de diferentes substratos ${ }^{35}$.

Tabela 3. BP's obtidos a partir de diferentes lactamas e compostos carbonílicos

\begin{tabular}{ll}
\hline Reagentes & Rendimento (\%) \\
\hline & \\
$\left(\mathrm{CH}_{3} \mathrm{CH}_{2}\right)_{2} \mathrm{~N}-\mathrm{C}-\mathrm{CH}_{3}$ & \\
\hline
\end{tabular}<smiles>[R]C/C=C(\CCC)CCC=C(C)C</smiles>

BP's derivados de $\alpha$-aminoácidos foram sintetizados, demonstrando também atividade anti-reabsortiva ${ }^{36}$. Nesta reação é feita, inicialmente, a proteção do grupo amina do aminoácido, seguida de substituição do grupo hidroxila pelo cloro. Posteriormente, é feita a inserção dos átomos de fósforo em duas etapas. Primeiramente, ocorre reação com um reagente adequado de fósforo, formando um fosfonato de acila e, em seguida, ocorre ataque de um segundo átomo de fósforo, formando o bisfosfonato. Finalmente, o grupo protetor é retirado, os grupos ésteres são hidrolisados e os BP's são obtidos como sais de sódio (Esquema 8) ${ }^{36}$.
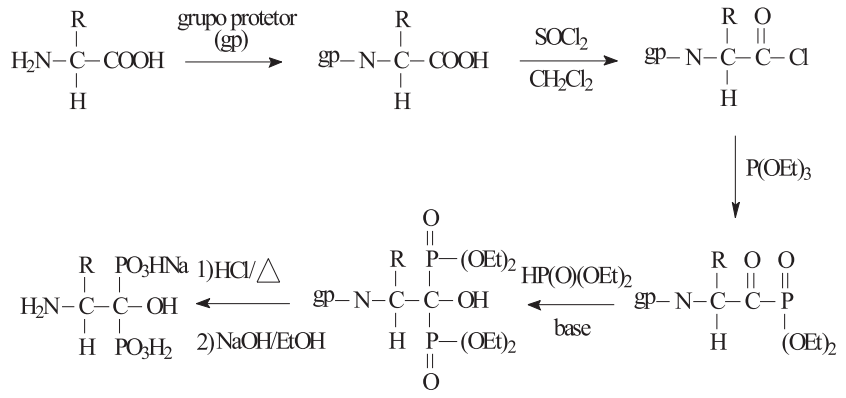

Esquema 8. Síntese de BP's derivados de $\alpha$-aminoácidos
Como descrito anteriormente, a estrutura da cadeia lateral, bem como a presença ou não de heteroátomo fornece características bem distintas aos BP's. O 2-aminociclopropilideno-1,1-bisfosfonato de tetraetila, ainda não avaliado clinicamente, possui anel de 3 membros em sua cadeia lateral, podendo proporcionar uma atividade farmacológica ainda não encontrada em outros $\mathrm{BP}^{3} \mathrm{~s}^{37}$.

A síntese deste composto é realizada em três passos (Esquema 9). Primeiramente, o lítiocarbânion do 2-bromoacetato de etila é adicionado, via adição de Michael, ao vinilideno 1,1-bisfosfonato de tetraetila. Essa adição é seguida de uma ciclização intramolecular levando à formação de um derivado ciclopropil. Posteriormente, este derivado é saponificado, e o ácido obtido é convertido em uma amina ${ }^{37}$.

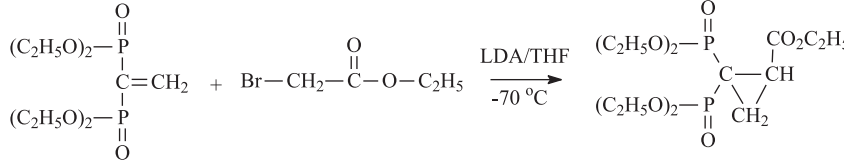

$$
\begin{aligned}
& \mathrm{KOH} \\
& \mathrm{H}_{2} \mathrm{O} / \mathrm{THF}
\end{aligned}
$$

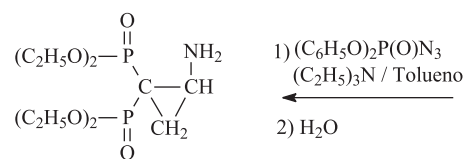

$$
\begin{aligned}
& \left(\mathrm{C}_{2} \mathrm{H}_{5} \mathrm{O}\right)_{2}-\stackrel{\mathrm{O}}{\mathrm{O}} \stackrel{\mathrm{P}}{\mathrm{P}} \stackrel{\mathrm{C}}{\mathrm{C}} \mathrm{CO}_{2} \mathrm{H}
\end{aligned}
$$

Esquema 9. Síntese do 2-aminociclopropilideno-1,1-bisfosfonato de tetraetila

\section{ANÁLISE}

Os BP's podem ser analisados por várias técnicas analíticas, destacando-se a cromatografia gasosa, a cromatografia líquida de alta eficiência e a eletroforese capilar. Estas técnicas são utilizadas para a determinação de BP's na matéria-prima, no produto acabado, e em fluidos biológicos, sendo neste caso necessário o desenvolvimento de metodologias analíticas com alta sensibilidade, tendo em vista a baixa concentração destes fármacos neste meio.

A análise em meio biológico requer, muitas vezes, a utilização de técnicas de pré-concentração, tais como microextração em fase sólida (SPME) e extração em fase sólida (SPE), sendo esta última a mais comum.

O desenvolvimento de métodos sensíveis é dificultado pelas características das moléculas dos BP's mais utilizados. Estes não possuem grupos cromóforos e não podem ser determinados pelos detectores mais usuais (ultravioleta e fluorescência). Para contornar esta dificuldade têm sido desenvolvidos métodos de derivação e detecção indireta. Entretanto, estes métodos requerem maior tempo para sua execução, além de serem mais passíveis de erros.

Nos últimos anos tem-se observado um aumento no desenvolvimento de métodos que utilizam a espectrometria de massas. Este detector possui a grande vantagem de ser seletivo e universal, além de possuir boa sensibilidade e robustez. Além disso, permite a análise direta, sem necessidade de derivação. Por outro lado, é uma técnica mais cara e que exige maior experiência por parte dos analistas.

\section{Cromatografia Líquida de Alta Eficiência (HPLC)}

Esta técnica é a mais utilizada para análise dos BP's, sendo empregados diferentes detectores, fases móveis e mecanismos de retenção.

Chester ${ }^{38}$ desenvolveu um detector seletivo para fósforo, baseado na emissão molecular de luz pelo HPO, para HPLC, analisando 
diversos ácidos fosfônicos. Posteriormente, o mesmo autor utilizou esse detector para análise do clodronato em fluidos biológicos. $\mathrm{O}$ mecanismo de separação empregado foi de troca aniônica, obtendose um limite de quantificação de $2 \mu \mathrm{mol} / \mathrm{L}^{39}$.

Foram relatados vários métodos utilizando detecção por índice de refração. Um método para determinação do ácido metilenobisfosfônico, utilizando pareamento iônico, foi desenvolvido por $\mathrm{Yeh}^{40}$. Foi utilizada uma coluna convencional $\mathrm{C}_{18}$ e o composto tri- $n$ octilamina como agente de pareamento iônico. Wong e colaboradores ${ }^{41}$ desenvolveram um método empregando coluna de troca aniônica, para análise de diferentes polifosfonatos, obtendo limites de detecção de 2,4 a 15,2 ppm. Han e colaboradores ${ }^{42}$ analisaram comprimidos de alendronato sódico utilizando coluna de troca aniônica. Este método mostrou-se exato, específico, robusto e com limite de detecção adequado para análises de rotina de comprimidos.

A análise de BP's utilizando detectores de fluorescência também é muito utilizada. Como estas moléculas, em geral, não possuem grupos que fluorescem, são necessárias algumas estratégias para a utilização de tais detectores. Derivatizações pré ou pós-coluna e detecção indireta são as mais utilizadas. No primeiro caso, uma molécula fluorescente é ligada ao BP, enquanto na detecção indireta o composto fluorescente encontra-se presente na fase móvel.

Métodos de determinação indireta de BP's foram desenvolvidos utilizando o complexo de alta fluorescência formado entre o alumínio e o morin (2',3', 4', 5,7-pentidroxiflavona). Na presença dos BP's o alumínio liga-se a estes compostos formando um complexo mais estável e que não fluoresce. A passagem do complexo alumínio-morin pelo detector de fluorescência gera absorção ao passo que o complexo BP-alumínio, ao passar pelo detector, causa diminuição da absorção, registrando um pico negativo ${ }^{43,44}$. A detecção por fluorescência com derivatização pós-coluna foi descrita por Kwong e colaboradores $^{45}$ para a análise de comprimidos de alendronato. Foram utilizados os compostos $o$-ftalaldeído e mercaptoetanol (OPA-MERC) como reagente pós-coluna, sendo avaliada a eficiência da reação de derivatização, variando-se os seguintes parâmetros: $\mathrm{pH}$, fluxo da fase móvel, fluxo do reagente pós-coluna, temperatura da reação, tamanho e tipo do reator. Foi utilizada uma coluna de fase reversa com pareamento iônico.

Vários compostos podem ser utilizados para derivatização précoluna em detecção por fluorescência. O pamidronato foi determinado em urina, empregando a fluorescamina como reagente de fluorescência e coluna de fase reversa $\left(\mathrm{C}_{18}\right)$. $\mathrm{O}$ limite de quantificação obtido foi de $1 \mu \mathrm{mol} / \mathrm{L}$ de urina e o limite de detecção foi de $50 \mathrm{nmol} /$ L de urina ${ }^{46}$. Posteriormente, foram alcançados melhores limites de quantificação e detecção $(0,7 \mu \mathrm{mol} / \mathrm{L}$ de urina e $10 \mathrm{nmol} / \mathrm{L}$ de urina, respectivamente), aprimorando-se o processo de extração dos analitos ${ }^{47}$.

O composto 2,3-naftaleno dicarboxaldeído também é utilizado em derivatizações pré-coluna. Kline e colaboradores ${ }^{48}$ determinaram o alendronato em urina utilizando este reagente, na presença do íon cianeto, e coluna de fase reversa polimérica. Foi obtido limite de quantificação de $5 \mathrm{ng} / \mathrm{mL}$. Este método foi melhorado substituindose o íon cianeto pelo composto $\mathrm{N}$-acetil-D-penicilamina, obtendo-se um limite de quantificação de $1 \mathrm{ng} / \mathrm{mL}^{49}$.

Outra opção para a derivatização pré-coluna é a utilização do reagente 1-naftilisotiocianato, para a determinação do pamidronato em fluidos biológicos. Para tanto, a separação foi feita por meio de pareamento iônico ${ }^{50-52}$.

Para a determinação de BP's utilizando detectores de ultravioleta (UV) são também necessárias estratégias, devido à ausência de grupos cromóforos nestes fármacos. Sparidans e colaboradores ${ }^{53}$ desenvolveram um método para a análise de oito diferentes BP's, onde estes são complexados "in-line" com íons cobre(II), absorvendo no UV em $245 \mathrm{~nm}$. Utilizou-se coluna de troca aniônica e fase móvel constituída de ácido nítrico $1,5 \mathrm{mmol} / \mathrm{L}$ e nitrato de cobre(II) 0,5 $\mathrm{mmol} / \mathrm{L}$. O limite de detecção obtido foi de $0,4 \mu \mathrm{g} / \mathrm{mL}^{53}$.

Foram também desenvolvidos métodos utilizando derivatizações

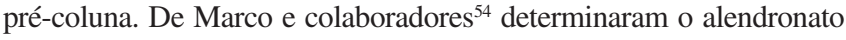
empregando o composto cloroformato de 9-fluorenilmetil (FMOC), na presença de citrato de sódio, como reagente de derivatização. Utilizou-se coluna de fase reversa polimérica, com detecção em 266 nm. Outro método foi desenvolvido utilizando o isotiocianato de fenila seguido de tratamento com peróxido de hidrogênio, antes da injeção. Os limites de detecção e quantificação obtidos foram de $0,1 \mu \mathrm{g} / \mathrm{mL}^{55}$.

A análise de BP's com detecção no UV-Visível e derivação póscoluna é muito utilizada. Daley-Yates e colaboradores ${ }^{56}$ desenvolveram um método para análise em plasma e urina. Os BP's foram oxidados a ortofosfato com persulfato de amônio e posterior reação com molibdênio-ascorbato, formando o cromóforo fosfomolibdato, detectado em $820 \mathrm{~nm}$. Obteve-se limite de detecção de $10 \mathrm{ng} / \mathrm{mL}$.

Foi desenvolvido outro método para análise de clodronato, empregando coluna de poliestireno-divinilbenzeno. $\mathrm{O}$ analito foi detectado em $300 \mathrm{~nm}$ após reação com solução ácida de ferro (III) ${ }^{57}$. Foram propostos métodos utilizando o complexo tório-EDTA-laranja de xilenol. Os BP's reagem quantitativamente com este complexo em condições levemente ácidas ${ }^{58,59}$

Outra alternativa para a detecção no UV são os métodos indiretos. Tsai e colaboradores ${ }^{60}$ propuseram um método para análise de comprimidos de etidronato utilizando ácido nítrico (absorção máxima em $220 \mathrm{~nm}$ ) como eluente. Os cromatogramas obtidos apresentam uma queda no sinal quando ocorre a passagem dos BP's pela célula do detector.

Detectores eletroquímicos também podem ser utilizados na determinação de BP's, com a vantagem de não haver necessidade de derivação do analito. Entretanto, são muito sensíveis a variações externas, o que compromete a reprodução dos resultados. Usui e colaboradores $^{61}$ propuseram um método para análise de um novo BP, extraído de plasma, urina e osso, empregando detector eletroquímico operando no modo oxidativo. Foram obtidos limites de quantificação de $0,5 \mathrm{ng} / \mathrm{mL}$ no plasma, $1 \mathrm{ng} / \mathrm{mL}$ na urina e $25 \mathrm{ng} / \mathrm{g}$ no osso.

Detectores condutimétricos são utilizados na determinação de diferentes BP's, também sem a necessidade de derivatização do analito. São empregadas colunas de troca aniônica ${ }^{62-64}$.

Qin e colaboradores ${ }^{65}$ desenvolveram método para a determinação de alendronato (altamente polar e termolábil) em comprimidos utilizando cromatografia iônica acoplada a detector de massas, empregando interface do tipo íon-spray, operando no modo negativo. Observou-se neste estudo que a fragmentação dominante deste composto é a clivagem das ligações C-P.

O clodronato e BP's relacionados foram analisados por espectrometria de massas com fonte de ionização por eletrospray no modo negativo, em um equipamento do tipo triplo quadrupolo. Foram propostos diferentes caminhos para a fragmentação destes compostos e avaliada a influência do $\mathrm{pH}$ no grau de desprotonação ${ }^{66}$.

\section{Cromatografia Gasosa}

Os BP's possuem natureza polar e baixa volatilidade. Desta forma, para análise por cromatografia gasosa, é necessário o processo de derivatização para a formação de compostos voláteis e estáveis. Comprimidos de etidronato foram analisados por meio da derivatização com bis(trimetilsilil)-trifluoroacetamida. Utilizou-se detector de ionização de chama para análise e detector de massas para controle do derivado formado. O método mostrou-se rápido e específico, porém sem sensibilidade suficiente para análise deste fármaco em fluidos biológicos ${ }^{67}$.

Auriola e colaboradores ${ }^{68}$ descreveram um método para análise 
de clodronato em urina. $\mathrm{O}$ analito extraído reagiu com um derivado trimetilsilil e foi analisado utilizando detector de massas.

Foi desenvolvida metodologia para análise de diferentes BP's nitrogenados, utilizando o composto cloroformato de isobutila como reagente de derivatização. Utilizou-se detector fotométrico de chama, obtendo-se limite de detecção no plasma de 200 ng/mL. Realizou-se também análise com espectrômetro de massas para confirmação estrutural dos derivados formados ${ }^{69}$.

\section{Eletroforese Capilar}

Isotacoforese capilar foi utilizada para análise de pamidronato e impurezas de síntese e degradação presentes. Foram comparados dois sistemas com diferentes condições e estes mostraram-se adequados para análises de rotina ${ }^{70}$.

Um método para análise de alendronato em formulações farmacêuticas foi descrito por Tsai e colaboradores ${ }^{71}$. Utilizou-se detecção direta no UV baseada na formação "on-line" de complexo cromóforo entre este fármaco e os íons cobre(II) presentes na solução eletrolítica. No entanto, este método não pode ser utilizado para análise de soluções intravenosas devido à presença dos ânions citrato (forte agente quelante) em suas formulações.

Sirén e colaboradores ${ }^{72}$ desenvolveram método para análise de BP's, com detecção indireta no UV, utilizando corantes nitrosonaftóis sulfonatados na solução eletrolítica. O limite de detecção obtido foi de $1 \mu \mathrm{g} / \mathrm{mL}$ em $254 \mathrm{~nm}$.

Peng e colaboradores ${ }^{73}$ determinaram um BP, 2-tioetano-1,1-ácido bisfosfônico, utilizando método com determinação direta no UV, coluna revestida com glicerol, fosfato em alta concentração como tampão e injeção eletrocinética sob condições de "sample stacking". O limite de detecção foi de $50 \mathrm{ng} / \mathrm{mL}$.

Um método para determinação de clodronato e suas impurezas, utilizando detecção de massas por eletrospray e modo negativo, foi desenvolvido por Huikko e colaboradores ${ }^{74}$. Este mostrou alta capacidade de separação e especificidade para análise de BP's.

Um método com detecção direta no UV em baixo comprimento de onda e polaridade invertida foi aplicado para separação e quantificação de impurezas em clodronato. Utilizou-se capilar de poliacrilamida e avaliou-se a influência do $\mathrm{pH}$ e força iônica do eletrólito sobre a separação ${ }^{75}$.

\section{Outros métodos}

Reed e colaboradores ${ }^{76}$ descreveram um método direto para análise de alendronato em comprimidos. As amostras foram dissolvidas em água e o conteúdo de fósforo foi analisado por plasma acoplado indutivamente (ICP). O método apresentou boa precisão e exatidão.

A formação de complexo entre alendronato e cloreto férrico em solução de ácido perclórico foi estudada e analisada por espectrofotometria. Este método apresentou limite de detecção de $2 \mu \mathrm{g} / \mathrm{mL}$, suficiente para análise nas formulações farmacêuticas ${ }^{77}$.

\section{CONCLUSÃO}

Os BP's são fármacos de escolha para uma variedade de doenças ósseas, destacando-se dentre estas a osteoporose, que tem se tornado nos últimos anos um importante problema de saúde pública, principalmente devido ao aumento da expectativa de vida da população.

Além disso, outras aplicações relevantes têm sido relatadas com o uso dos BP's. Estes fármacos têm se mostrado ativos sobre o metabolismo de vários protozoários, podendo ser utilizados no tratamento da doença de Chagas, leishmaniose visceral, malária e toxoplasmose, doenças que acometem milhões de indivíduos em todo o mundo.
Um grande número de rotas sintéticas tem sido relatado para a preparação de diferentes BP's, objetivando-se melhorar as condições experimentais, os rendimentos e os custo, bem como encontrar estruturas com maior potência e atividade farmacológica.

Os BP's, devido à sua baixa absorção, são encontrados em concentrações muito pequenas nos fluidos biológicos. A grande maioria dos métodos analíticos descritos mostraram-se adequados para a determinação destes fármacos na matéria-prima e nas formulações farmacêuticas, entretanto, ainda há a necessidade do desenvolvimento de métodos mais sensíveis, de fácil execução e de baixo custo, para determinações em meio biológico.

Métodos adequados de análise em fluidos biológicos são fundamentais para a realização de estudos de biodisponibilidade e bioequivalência. Este último constitui uma das etapas na obtenção de um medicamento genérico, que é atualmente considerada como política de prioridade do Ministério da Saúde do Brasil.

\section{AGRADECIMENTOS}

Os autores agradecem à CAPES, CNPq e FAPESP pelo apoio e concessão de bolsas.

\section{REFERÊNCIAS}

1. Licata, A. A.; Am. J. Med. Sci. 1997, 313, 17.

2. Fleisch, H.; Recent Results Cancer Res. 1989, 116, 1.

3. Shinoda, H.; Adamek, G.; Felix, R.; Fleisch, H.; Schenk, R.; Hagan, P.; Calcif. Tissue Int. 1983, 35, 87.

4. Fleisch, H. Em Bone and mineral metabolism; Fleisch, H., ed.; Stampfli: Berne, 1993, p. 8.

5. Neves, M.; Gano, L.; Pereira, N.; Costa, M. C.; Costa, M. R.; Chandia, M.; Rosado, M.; Fausto, R.; Nucl. Med. Biol. 2002, 29, 329.

6. Sahni, M.; Guenther, H. L.; Fleisch, H.; Collin, P.; Martin, T. J.; J. Clin. Invest. 1993, 91, 2004.

7. Green, J. R.; Breast Cancer Res. 2000, 3, A28.

8. Lin, J. H.; Bone 1996, 18, 75.

9. Fleisch, H.; Endocrine Rev. 1998, 19, 80.

10. Reitsma, P. H.; Dissertação de mestrado, Universidade de Leiden, Holanda, 1982.

11. Szajnman, S. H.; Bailey, B. N.; Docampo, R.; Rodriguez, J. B.; Bioorg. Med. Chem. Lett. 2001, 11, 789.

12. Martin, M. B.; Grimley, J. S.; Lewis, J. C.; Heath, H. T.; Bailey, B. N.; Kendrick, H.; Yardley, V.; Caldera, A.; Lira, R.; Urbina, J. A.; Moreno, S. N. J.; Docampo, R.; Croft, S. L.; Oldfield, E.; J. Med. Chem. 2001, 44, 909.

13. Nugent, R. A.; Murphy, M.; Schlachter, S. T.; Dunn, C. J.; Smith, R. J.; Staite, N. D.; Galinet, L. A.; Shields, S. K.; Wu, H.; Aspar, D. G.; Richard, K. A.; Rohloff, N. A.; J. Med. Chem. 1993, 36, 134.

14. Nugent, R. A.; Schlachter, S. T.; Murphy, M.; Dunn, C. J.; Staite, N. D.; Galinet, L. A.; Shields, S. K.; Wu, H.; Aspar, D. G.; Richard, K. A.; J. Med. Chem. 1994, 37, 4449.

15. Kontecka, E. G.; Silvagni, R.; Lipinski, R.; Lecouvey, M.; Marincola, F. C.; Crisponi, G.; Nurchi, V. M.; Leroux, Y.; Kozlowski, H.; Inorg. Chim. Acta 2002, 339, 111.

16. Rodrigues, J. M.; DaCosta, J. B. N.; Phosphorus, Sulfur Silicon Relat. Elem. 2002, 177, 137.

17. Nixon, G. A.; Buehler, E. V.; Newmann, E. A.; Toxicol. Appl. Pharmacol. 1972, 22, 661 .

18. Flora, L.; Hassing, G. S.; Parfitt, A. M.; Villanueva, A. R.; Metab. Bone Disease Relat. Res. 1980, 2, 389.

19. Singer, F. R.; Ritch, P. S.; Lad, T. E.; Ringeberg, Q. S.; Schiller, J. H.; Archives of Internal Medicine 1991, 151, 471.

20. Markkula, R.; Repo, H.; Leirisalo, M.; Blomqvist, C.; Elomaa, I.; Cancer Immunol. Immunother. 1983, 15, 159.

21. Dodwell, D. J.; Howell, A.; Ford, J.; Br. J. Cancer 1990, 61, 123.

22. Gibaldi, M.; Perrier, D. Em Pharmacokinetics; Gibaldi, M.; Perrier, D., eds.; Marcel Dekker: New York, 1982, $2^{\text {nd }}$ ed., p. 145-198.

23. Lin, J. H; Chen, I-W.; deLuna, F. A.; J. Pharm Sci. 1994, 83, 1741.

24. Ruifrok, P. G.; Mol, W. E. M.; Biochem. Pharmacol. 1983, 32, 637.

25. Gertz, B. J.; Holland, S. D.; Kline, W. F.; Matuszewski, B. K.; Porras, A. G.; Osteopor. Int. 1993, 3 (suppl.3), 513.

26. Lin, J. H.; Chen, I-W; deLuna, F. A.; Hichens, M.; J. Pharmacol. Exp. Ther. 1993, 267, 670. 
27. Michael, W. R.; King, W. R.; Wakin, J. M.; Pharmacol. Toxicol. 1988, 62, 51.

28. Wada, H.; Fernando, Q.; Anal. Chem. 1971, 43, 751.

29. Fleisch, H.; Drugs 1991, 42, 919.

30. Kieczykowski, G. R.; Jobson, R. B.; Melillo, D. G.; Reinhold, D. F.; Grenda, V. J.; Shinkai, I.; J. Org. Chem. 1995, 60, 8310.

31. Lecouvey, M.; Mallard, I.; Bailly, T.; Burgada, R.; Leroux, Y.; Tetrahedron Lett. 2001, 42, 8475 .

32. Vogel, A. I.; A textbook of practical organic chemistry including qualitative organic analysis, Longmans: London, 1951.

33. Allen, A. J.; Manke, D. R.; Lin, W.; Tetrahedron Lett. 2000, 41, 151.

34. McCombie, H.; Saunders, B. C.; Stacey, G. J.; J. Chem. Soc. 1945, 380

35. Du, Y.; Jung, K. Y.; Wiemer, D. F.; Tetraheron Lett. 2002, 43, 8665.

36. Mizrahi, D. M.; Waner, T.; Segall, Y.; Phosphorus, Sulfur Silicon Relat. Elem. 2001, 173, 1.

37. Couthon, H.; Gourvès, J. P.; Guervenou, J.; Corbel, B.; Sturtz, G.; Synth. Commun. 1999, 29, 4251.

38. Chester, T. L.; Anal. Chem. 1980, 52, 1621.

39. Chester, T. L.; Lewis, E. C.; Benedict, J. J.; Sunberg, R. J.; Tettenhorst, W. C.; J. Chromatogr. 1981, 225, 17.

40. Yeh, P.; J. Chromatogr. Sci. 1981, 19, 27.

41. Wong, D.; Jandik, P.; Jones, W. R.; Hagenaars, A.; J. Chromatogr. 1987, 389, 279.

42. Han, Y-H. R.; Qin, X-Z.; J. Chromatogr., A 1996, 719, 345

43. Meek, S. E.; Pietrzyk, D. J.; Anal. Chem. 1988, 60, 1397.

44. Lovdahl, M. J.; Pietrzyk, D. J.; J. Chromatogr., A 1999, 850, 143.

45. Kwong, E.; Chiu, A. M. Y.; McClintock, S. A.; Cotton, M. L.; J. Chromatogr. Sci. 1990, 28, 563.

46. Flesch, G.; Hauffe, S. A.; J. Chromatogr. 1989, 489, 446.

47. Flesch, G.; Tominaga, N.; Degen, P.; J. Chromatogr. 1991, 568, 261.

48. Kline, W. F.; Matuszewski, B. K.; Bayne, W. F.; J. Chromatogr. 1990, 534, 139.

49. Kline, W. F.; Matuszewski, B. K.; J. Chromatogr. 1992, 583, 183.

50. Sparidans, R. W.; Hartigh, J. D.; Beijnen, J. H.; Vermeij, P.; J. Chromatogr., A 1997, 782, 211.

51. Sparidans, R. W.; Hartigh, J. D.; Beijnen, J. H.; Vermeij, P.; J. Chromatogr., B: Biomed. Sci. Appl. 1997, 696, 137.

52. Sparidans, R. W.; Hartigh, J. D.; Beijnen, J. H.; Vermeij, P.; J. Chromatogr., B: Biomed. Sci. Appl. 1998, 705, 331.

53. Sparidans, R. W.; Hartigh, J. D.; Vermeij, P.; J. Pharm. Biomed. Anal. 1995, $13,1545$.
54. De Marco, J. D.; Biffar, S. E.; Reed, D. G.; Brooks, M. A.; J. Pharm. Biomed. Anal. 1989, 7, 1719.

55. Sparidans, R. W.; Hartigh, J. D.; Ramp-Koopmanschap, W. M.; Langebroek, R. H.; Vermeij, P.; J. Pharm. Biomed. Anal. 1997, 16, 491.

56. Daley-Yates, P. T.; Gifford, L. A.; Hoggarth, C. R.; J. Chromatogr. 1989, 490, 329.

57. Kosonen, J. P.; J. Pharm. Biomed. Anal. 1992, 10, 881.

58. Virtanen, V.; Lajunen, L. H. J.; J. Chromatogr. 1993, 617, 291.

59. Virtanen, V.; Lajunen, L. H. J.; Talanta 1993, 40, 661.

60. Tsai, E. W.; Ip, D. P.; Brooks, M. A.; J. Pharm. Biomed. Anal. 1993, 11, 513.

61. Usui, T.; Watanabe, T.; Higuchi, S.; J. Chromatogr. 1992, 584, 213.

62. Tsai, E. W.; Ip, D. P.; Brooks, M. A.; J. Chromatogr. 1992, 596, 217.

63. Hartigh, J. D.; Langebroek, R.; Vermeij, P.; J. Pharm. Biomed. Anal. 1993, $11,977$.

64. Taylor, G. E.; J. Chromatogr., A 1997, 770, 261.

65. Qin, X-Z.; Tsai, E. W.; Sakuma, T.; Ip, D. P.; J. Chromatogr., A 1994, 686, 205 .

66. Huikko, K.; Kotiaho, T.; Yli-Kauhaluoma, J.; Kostiainen, R.; J. Mass Spectrom. 2002, 37, 197.

67. Ismail, Z.; Aldous, S.; Triggs, E. J.; Smithurst, B. A.; Barry, H. D.; J. Chromatogr. 1987, 404, 372.

68. Auriola S.; Kostiainen, R.; Ylinen, M.; Mönkkonen, J.; Ylitalo, P.; J. Pharm. Biomed. Anal. 1989, 7, 1623.

69. Sakyiama, N.; Kataoka, H.; Makita, M.; J. Chromatogr., A 1996, 724, 279.

70. Zeller, M.; Kessler, R.; Manz, H. J.; Székely, G.; J. Chromatogr. 1991, 545, 421.

71. Tsai, E. W.; Singh, M. M.; Lu, H. H.; Ip, D. P.; Brooks, M. A.; J. Chromatogr. 1992, 626, 245.

72. Sirén, H.; Määttänen, A.; Riekkola, M. L.; J. Chromatogr., A 1997, 767, 293.

73. Peng, S. X.; Takigiku, R.; Burton, D. E.; Powell, L. L.; J. Chromatogr., B: Biomed. Sci. Appl. 1998, 709, 157.

74. Huikko, K.; Kostiainen, R.; J. Chromatogr., A 2000, 872, 289.

75. Huikko, K.; Kostiainen, R.; J. Chromatogr., A 2000, 893, 411.

76. Reed, D. G.; Martin, G. P.; Konieczny, J. M.; Brooks, M. A.; J. Pharm. Biomed. Anal. 1995, 13, 1055.

77. Kuljanin, J.; Jancovic, I.; Nendeljkovic, J.; Prstojevic, D.; Marinkovic, V.; J. Pharm. Biomed. Anal. 2002, 28, 1215. 